\title{
Production of gelatin microparticles in a flow focusing microfluidic device for biomedical applications
}

\author{
A. I. Moreira' ${ }^{1}$ J. Carneiro ${ }^{1}$ J. B. L. M. Campos ${ }^{1}$, J. M. Miranda ${ }^{1}$ \\ ${ }^{1}$ CEFT, Transport Phenomena Research Center, Chemical Engineering Department \\ Faculty of Engineering, University of Porto, Rua Roberto Frias s/n, 4050-465 Porto, Portugal \\ aidm@fe.up.pt, jcarneiro@fe.up.pt; jmc@fe.up.pt; jmiranda@ @e.up.pt
}

\begin{abstract}
Particles that can mimetize some of the mechanical and geometrical properties of red blood cells and other cellular components of the human body can have important applications in the development of drug delivery carriers and blood substitutes. In this work gelatin microparticles are produced in a droplet microfluidics flow focusing device. Several operating conditions are studied and, for the best conditions, particles of 14-16 $\mu \mathrm{m}$ are obtained. Two flow regimes are observed, dripping, when the Capillary number of the dispersed phase is smaller than 0.01 , and jetting when the capillary number of the dispersed phase is higher than 0.01 .
\end{abstract}

Keywords: Droplet microfluidics, biomimetic particles, gelatin, drug carriers.

\section{Introduction}

Biomedical research requires the realistic in vitro mimetization of in vivo conditions to test medical devices, new drug delivery systems or to perform experiments to understand the response of tissues and organs. In some of these experiments, biological cells and tissues are used. However, not all experiments require living cells and tissues. In some cases the use of materials of biological origin have high gathering and maintenance costs. And in the case of humans, ethical issues limit the availability of tissues and cells. So, a demand exists for realistic mimics that can, at least in most experiments, substitute biological materials. Among the products that may be of interest are fluids that mimic the rheological properties of blood [1] and particles that mimic blood cells behaviour in microcirculation [2].

Hydrogels are a family of materials with a large variety of properties, applications and fabrication paths. Hydrogels are obtained by crosslinking from monomers in aqueous solutions. Polymer networks with the ability to absorb water are obtained. Crosslinking can be induced by different changes in conditions, such as changes in temperature, $\mathrm{pH}$ or addition of an initiator. Hydrogels are usually biocompatible and can be used as drug delivery carriers and cell encapsulation [3].

In this work we are concerned with the production micro sized particles that can be applied as drug carriers in the blood stream or in artificial microfluidic networks. The goal is to obtain monodispersed particles near the 6-10 $\mu \mathrm{m}$ range by droplet microfluidics. To achieve this objective, experiments are conducted to optimize the operational conditions.

\section{Methods}

\subsection{Device fabrication}

The experiments were conducted in PDMS microchannels obtained by soft lithography from SU-8 molds, following our previous work [4]. No plasma treatment was applied to the microchannel, and so the surface was kept hydrophobic. The design used is represented in Fig. 1. The dept of the microchannel is $50 \mu \mathrm{m}$, the width of the main channel is $50 \mu \mathrm{m}$ and the width of the narrow passage is $20 \mu \mathrm{m}$. The geometry contains arrays of pillars that function as pre-filters to avoid the clogging of the narrow gap of the main microchannel. 

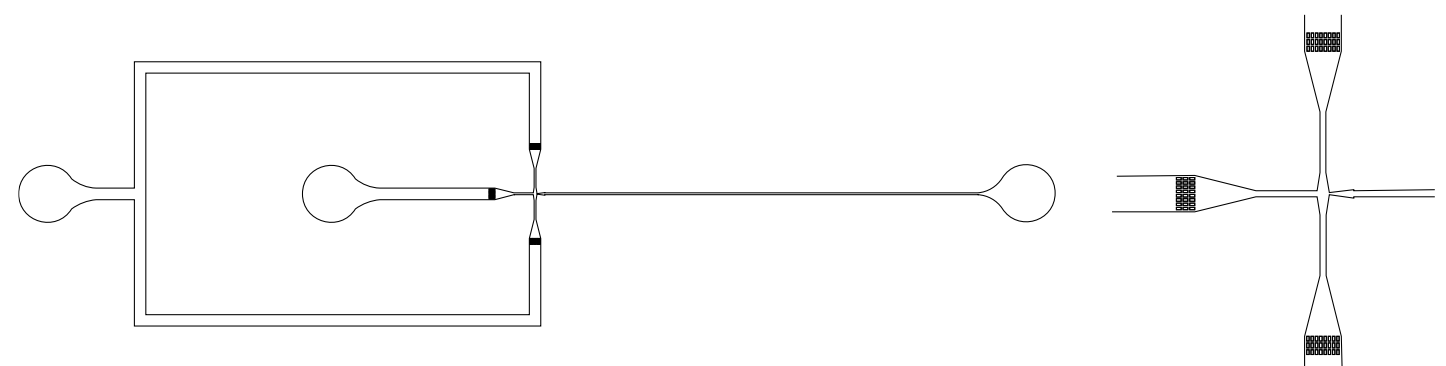

Fig. 1: Microfluidic device. Full view of the device (left) and detailed view of the intersection where the droplets form (right).

\subsection{Fluid properties}

The particles were produced by droplet microfluidics using mineral oil with 3\% of SPAN 80 as the continuous phase and a solution of $5 \%$ gelatin as the dispersed phase. The fluids properties are presented in Table 1.

Table 1: Fluids properties.

\begin{tabular}{|l|c|c|c|}
\hline \multicolumn{1}{|c|}{ Fluid } & $\begin{array}{c}\text { Viscosity } \\
(\text { Pa.s) }\end{array}$ & $\begin{array}{c}\text { Density } \\
\left(\mathbf{K g} / \mathbf{m}^{\mathbf{3}}\right)\end{array}$ & $\begin{array}{c}\text { Interfacial } \\
\text { tension (N/m) }\end{array}$ \\
\hline 5\% gelatin & 0.0050 & 1008.9 & 0.0038 \\
\cline { 1 - 2 } $\begin{array}{l}\text { Mineral oil }+3 \% \\
\text { Span 80 }\end{array}$ & 0.0151 & 854.1 & \\
\hline
\end{tabular}

\subsection{Droplet formation}

The gelatin was dissolved in hot water and subsequently cooled to $40^{\circ} \mathrm{C}$. The droplets were produced in the microfluidic device. Several flow rates were tested to test different flow conditions. The emulsion obtained was cooled at $5^{\circ}$ $\mathrm{C}$ to solidify the droplets and form particles. Images of droplet formation were obtained through an inverted microscope by a high speed camera, following a previous procedure [4].

\section{Results}

According to the literature, the flow pattern in flow focusing droplet microfluidics depends on the capillary numbers of the two phases. The Capillary numbers are defined as:

$$
\mathrm{Ca}_{c}=\frac{\mu_{c} v_{c}}{\sigma} ; \mathrm{Ca}_{d}=\frac{\mu_{d} v_{d}}{\sigma}
$$

where $\mu$ is the viscosity, $v$ the velocity, $\sigma$ the interfacial tension and $c$ and $d$ refer to the continuous and dispersed phases, respectively.

Additionally, it may also depend on the viscosity ratio and on the Weber numbers. The Weber numbers are defined as:

$$
\mathrm{We}_{c}=\frac{\rho_{c} v_{c}^{2} W}{\sigma} ; \mathrm{We}_{d}=\frac{\rho_{c} v_{d}^{2} W}{\sigma}
$$

where $\rho$ is the fluid density and $W$ is the width of the main channel.

Here it is assumed that the inertial effects are negligible since the Weber numbers are smaller than 0.05. The flow pattern map in the form of a diagram $\left(\mathrm{Ca}_{\mathrm{c}}, \mathrm{Ca}_{\mathrm{d}}\right)$ is shown in Fig. 2. The viscosity ratio is 0.33 . For $\mathrm{Ca}_{\mathrm{c}}$ lower than 0.01 , the flow is in the dripping regime while above 0.01 the flow is in the jetting regime for $\mathrm{Ca}_{\mathrm{d}}$ lower than 0.1 . Above 0.1 only one point is 
available, corresponding to dripping regime with the formation of multiple droplets. The size of the droplets is represented in Fig. 3.

The boundary between the dripping and the jetting regimes obtained for a viscosity ratio of 0.33 is similar to the one obtained in previous work [4] for a viscosity ratio of 533, suggesting that the regime map is independent of the viscosity ratio. However it will be necessary to obtain more data points to define the boundary more accurately.

The best operational conditions to obtain monodispersed small particles is $\mathrm{Ca}_{\mathrm{c}}=0.1$ and $\mathrm{Ca}_{\mathrm{d}}=0.019$. In this condition the particles have $16 \mu \mathrm{m}$. Smaller particles $(14 \mu \mathrm{m})$ can be obtained for $\mathrm{Ca}_{\mathrm{c}}>0.143$ and $\mathrm{Ca}_{\mathrm{d}}=0.019$, but in this case the particles are not monodispersed. Higher values of $\mathrm{Ca}_{\mathrm{d}}$ need to be explored, especially since the amount of particles produced is higher for higher flow rates of the dispersed phase, however above $\mathrm{Ca}_{\mathrm{d}}=0.019$ the flow is probably in the jetting regime and the droplets become increasingly polydispersed.

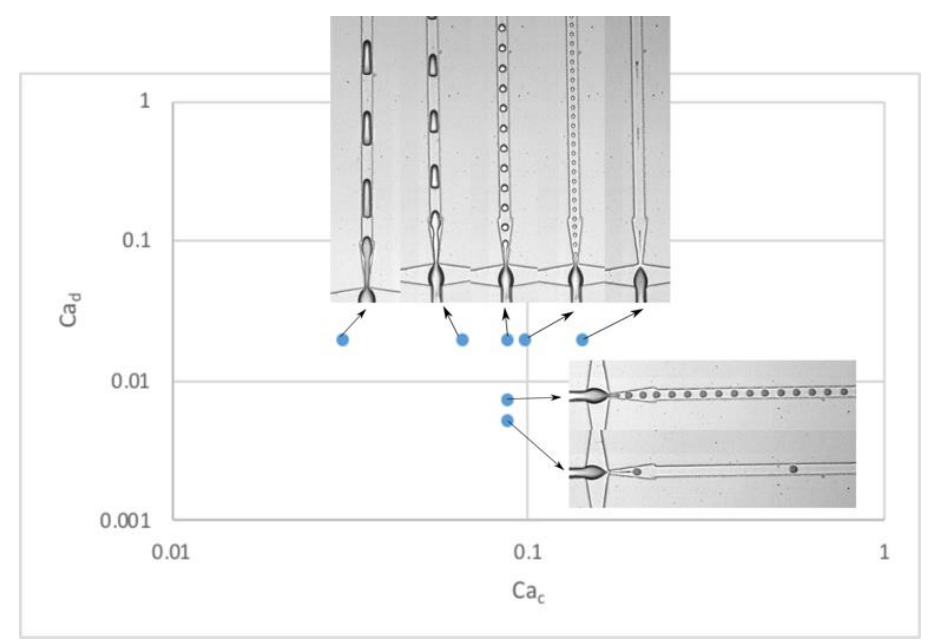

Fig. 2: Droplets formed for different operational conditions.

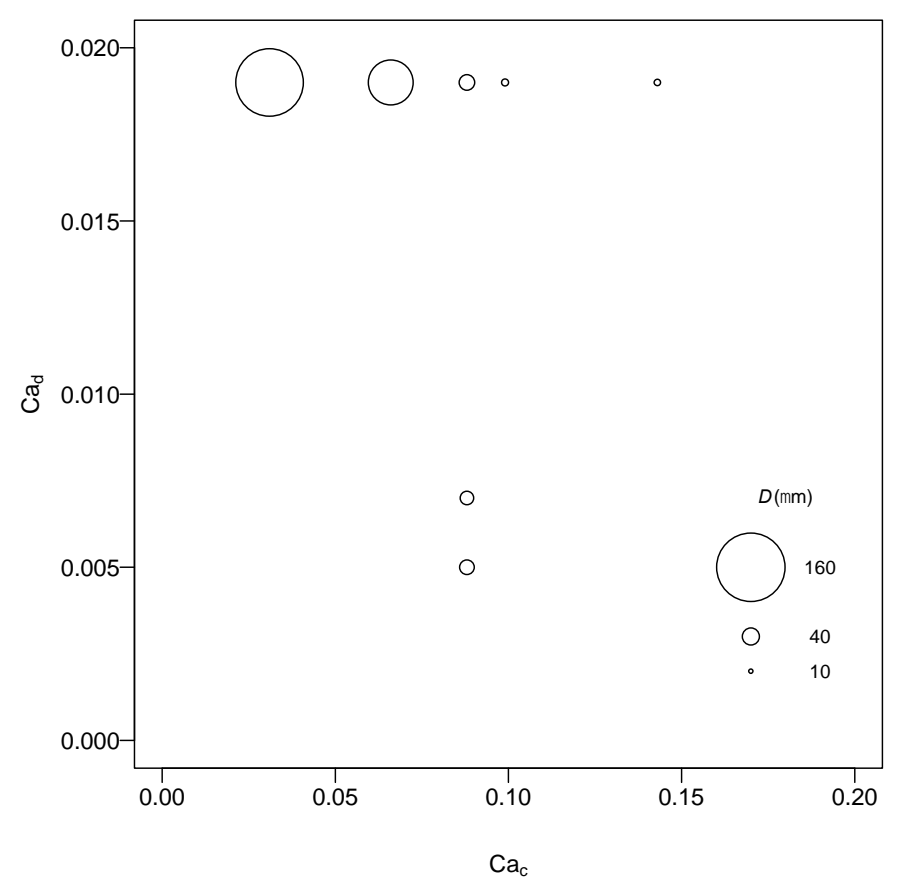

Fig. 3: Droplets size. Each data point is represented by a circle proportional to the size of the droplets. 


\section{Conclusion}

The formation of microparticles in a microfluidic device was studied for a set of operational conditions. It was found that the jetting regime appears when the Capillary number of the dispersed phase is above 0.01 . The regime map is consistent with the regime map obtained for much higher viscosity ratios. It was possible to produce monodispersed microparticles with $16 \mu \mathrm{m}$.

Further work is necessary to characterize the regime map with additional data points. Deformation tests in microfluidic channel constrictions will be conducted to characterize the mechanical properties of the particles and their behaviour in flow conditions. Redesign of the device may be necessary to reduce the size of the particles obtained.

\section{Acknowledgements}

This work was funded by FEDER funds through COMPETE2020-Operational Programme for Competitiveness Factors (POCI) and National Funds (PIDDAC) through FCT (Fundação para a Ciência e a Tecnologia) under projects PEstOE/EME/UI0532 and POCI-01-0145-FEDER-016861-PTDC/QEQ-FTT/4287/2014.

\section{References}

[1] L. Campo-Deaño, R. P. Dullens, D. G. Aarts, F. T. Pinho, and M. S. Oliveira, "Viscoelasticity of blood and viscoelastic blood analogues for use in polydymethylsiloxane in vitro models of the circulatory system," Biomicrofluidics, vol. 7, pp. 034102, 2013.

[2] T. J. Merkel, S. W. Jones, K. P. Herlihy, F. R. Kersey, A. R. Shields, M. Napier, et al., "Using mechanobiological mimicry of red blood cells to extend circulation times of hydrogel microparticles," in Proceedings of the National Academy of Sciences of the United States of America, 2011, vol. 108, pp. 586-591.

[3] C.-H. Choi, J.-H. Jung, Y. W. Rhee, D.-P. Kim, S.-E. Shim, and C.-S. Lee, "Generation of monodisperse alginate microbeads and in situ encapsulation of cell in microfluidic device," Biomedical Microdevices, vol. 9, pp. 855-862, 2007.

[4] J. Carneiro, E. Doutel, J. B. L. M. Campos, and J. M. Miranda, "PDMS droplet formation and characterization by hydrodynamic flow focusing technique in a PDMS square microchannel," Journal of Micromechanics and Microengineering, vol. 26, p. 105013, 2016. 